\title{
Nonclassical magnetic dynamics and negative exchange bias in $\mathrm{Nd}_{0.5} \mathrm{Sr}_{0.5} \mathrm{MnO}_{3}$ films
}

\author{
V.G. Prokhorov, G.G. Kaminsky, and V.A. Komashko \\ Institute of Metal Physics of the National Academy of Sciences of Ukraine, Kiev 03142, Ukraine \\ E-mail: pvg@imp.kiev.ua \\ Y.P. Lee, S.Y. Park, Y.H. Hyun, J.B. Kim, and J.S. Park \\ q-Psi and Department of Physics, Hanyang University, Seoul 133-791, Korea
}

V.L. Svetchnikov

National Center for HREM, TU Delft, 2628AL, The Netherlands

V.P. Pashchenko and V.A. Khokhlov

Donetsk Physical and Technical Institute of the National Academy of Sciences of Ukraine, Donetsk 83114, Ukraine

Received November 13, 2006, revised January 10, 2007

\begin{abstract}
The amorphous, nanoclustered, and self-organizing bilayered $\mathrm{Nd}_{0.5} \mathrm{Sr}_{0.5} \mathrm{MnO}_{3}$ films have been prepared by a rf-magnetron sputtering. The amorphous film turn out to be a typical paramagnet with a freely moving of the individual Mn spins, the magnetic properties of which are well described by the Curie-Weiss approximation. The nanoclustered film manifests the magnetic properties mimic to the superparamagnetic particles with a nonclassical magnetic dynamics. Taking into account the unique shape of the hysteresis loops, which have hysteretic lobes at high magnetic field but are nonhysteretic as the field crosses zero, we suggest that each particle (nanocluster) is the closure magnetic domain (or magnetic vortex) rather than the single one. At the same time, the blocked to unblocked transition was observed with increasing temperature similar to the usual superparamagnet. The self-organizing bilayered film demonstrates a negative exchange bias, which is typical for the ferromagnet/antiferromagnet hybrid system in spite of that both layers in our case have a ferromagnetic origin. The magnetic properties of the films are discussed in detail on the base of modern theoretical models.
\end{abstract}

PACS: 73.63.Bd Nanocrystalline materials;

75.47.Gk Colossal magnetoresistance;

75.47.Lx Manganites;

75.75.+a Magnetic properties of nanostructures.

Keywords: manganites, microstructure, magnetization.

\section{Introduction}

The hole-doped manganites $\mathrm{L}_{1-x} \mathrm{~A}_{x} \mathrm{MnO}_{3}$, where $\mathrm{L}$ and $\mathrm{A}$ are a trivalent lanthanide and a divalent alkaline-earth ions, respectively, have attracted considerable attention due to their interesting fundamental science and potential for applications [1]. Their peculiar physical behavior originates from the strong coupling between magnetic, structural, electronic, and orbital degrees of freedom and is manifested more greatly in the half-doped perovskite compounds. $\mathrm{Nd}_{1-x} \mathrm{Sr}_{x} \mathrm{MnO}_{3}$ is a typical system whose ground state varies from a ferromagnetic (FM) metal to an A-type antiferromagnetic (AFM) metal (or insulator at $x \geq 0.6$ ) when the Sr-doping crosses 0.5 [2]. In addition, the charge-ordered (CO) phase with the AFM CE-type structural is formed in the very narrow doping range $0.48 \leq x \leq 0.51$. The band structure calculations reveal that the energy difference between the FM and AFM phases is very small (around $0.01 \mathrm{eV} /$ atom) [3]. As consequence, the ground state of $\mathrm{Nd}_{0.5} \mathrm{Sr}_{0.5} \mathrm{MnO}_{3}$ is drastically 
dependent on the degree of crystallinity, the lattice strain, the chemical homogeneity, and the clustering of microstructure, which are controlled by a making technique. Thus, the $\mathrm{CO}$ state, for example, observes in the $\mathrm{Nd}_{0.5} \mathrm{Sr}_{0.5} \mathrm{MnO}_{3}$ film with a tensile in-plane strain [4], while does not find in that with a compressive one [5]. The CO AFM CE-type state can be suppressed by the increasing film thickness [6], the high hydrostatic pressure $[7,8]$, the decreasing grain size in polycrystalline samples [9], and the applied electrical field [10]. On the other hand, the influence of the structural quench disorder and the fine-crystalline clustering on the magnetic and charge ordering in this compound is still poorly understood.

In this paper, we report the experimental results for the $\mathrm{Nd}_{0.5} \mathrm{Sr}_{0.5} \mathrm{MnO}_{3}$ films with the different fine-crystalline microstructure. The observed evidence for the magnetic vortex state and the negative exchange bias effect are discussed in detail.

\section{Experimental techniques}

The $\mathrm{Nd}_{0.5} \mathrm{Sr}_{0.5} \mathrm{MnO}_{3}$ films were prepared by the rf-magnetron sputtering using a so-called «soft» (or powder) target [11]. The substrate was a $\mathrm{LaAlO}_{3}(001)$ single crystal (LAO) with an out-of-plane lattice parameter $c \simeq 0.379 \mathrm{~nm}$ for the pseudocubic symmetry. The substrate temperature $\left(T_{\text {sub }}\right)$ during deposition was varied from 20 to $800{ }^{\circ} \mathrm{C}$, and only the as-deposited films were used for a study. The thickness of all the films was $\simeq 100 \mathrm{~nm}$. The $\theta-2 \theta \mathrm{x}$-ray diffraction (XRD) patterns were obtained using a Rigaku diffractometer with $\mathrm{Cu} K_{\alpha}$ radiation. The lattice parameters evaluated directly from the XRD data were plotted against $\cos ^{2} \theta / \sin \theta$. From the intercept of the extrapolated straight line to $\cos ^{2} \theta / \sin \theta=0$, a more precise lattice parameter was obtained. The high-resolution electron-microscopy (HREM) and electron-diffraction (ED) studies were carried out using a Philips CM300UT-FEG microscope with a field emission gun operated at $300 \mathrm{kV}$. The point resolution of the microscope was in the order of $0.12 \mathrm{~nm}$. The cross-sectional specimens were prepared by the standard techniques using mechanical polishing followed by ion-beam milling at a grazing incidence. The magnetic measurements were performed by using a Quantum Design SQUID magnetometer.

\section{Microstructure of films}

Figure 1 presents the $\theta-2 \theta$ XRD scans for the films deposited at $T_{\text {sub }} \simeq 20(a), 300(b)$ and $750(c){ }^{\circ} \mathrm{C}$, respectively. The film deposited at the lowest substrate temperature manifests the very fuzzy Bragg peaks near (00l)-like reflections of LAO-substrate only that is typical for fully disordered or amorphous crystal structure [12]. Inset in Fig. 1, $a$ displays the fast Fourier transform (FFT) of the

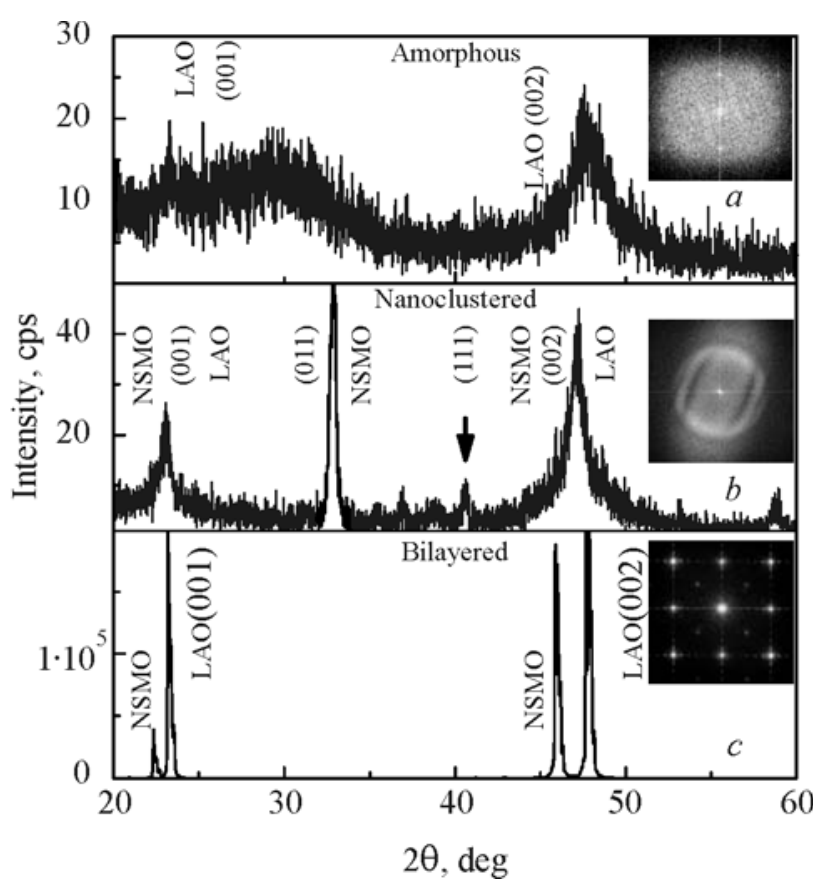

Fig. 1. $\theta-2 \theta$ XRD scans of amorphous (a), nanoclustered (b), and bilayered (c) films. Insets are FFT of the corresponding HREM images.

cross-sectional HREM image across the film/substrate interface. It is seen that FFT produces a bright uniform halo instead of a rectangular pattern of circular spots. Only the slightly luminescent spots corresponding to a crystal lattice of substrate are just-noticeable. Therefore, one can conclude that the film deposited at $T_{\text {sub }} \simeq 20{ }^{\circ} \mathrm{C}$ reveals an amorphous structure.

The film deposited at middle- $T_{\text {sub }}$ manifests the $(0 \mathrm{kl})$ and $(h k l)$-like Bragg peaks in addition to the broad (00l) XRD reflections. The FFT pattern, represented by the inset in Fig. 1,b, shows a wide ring, which is typical for a nano-scale disordered crystal structure [12]. Additional ED analysis (no shown) reveals that the average size of the randomly-oriented crystallites turn out to be about $4 \mathrm{~nm}$. This film hereinafter will be referred as a nanoclustered one.

The more unexpected microstructure was found for the film grown at $T_{\text {sub }} \simeq 750^{\circ} \mathrm{C}$. XRD scan displays the (00l) Bragg peaks of high intensity only, indicating that the deposition results in the highly $c$-oriented microstructure. The FFT of cross-sectional HREM image produces mainly a rectangular pattern of spots that is typical for a perfect crystal lattice. It is confirmed by the analysis of the ED pattern prepared at room temperature (see inset in Fig. 2,a), which reveals the well-known orthorhombic crystal structure with the lattice parameters for a simple pseudocubic symmetry about $a \simeq b \simeq 0.3852 \mathrm{~nm}$ and $c \simeq 0.3856 \mathrm{~nm}$, respectively, that are almost coincident with the bulk $[2,8,13]$. Figure $2, b$ shows that the ED pattern prepared at $87 \mathrm{~K}$ is the same. On the other hand, a 


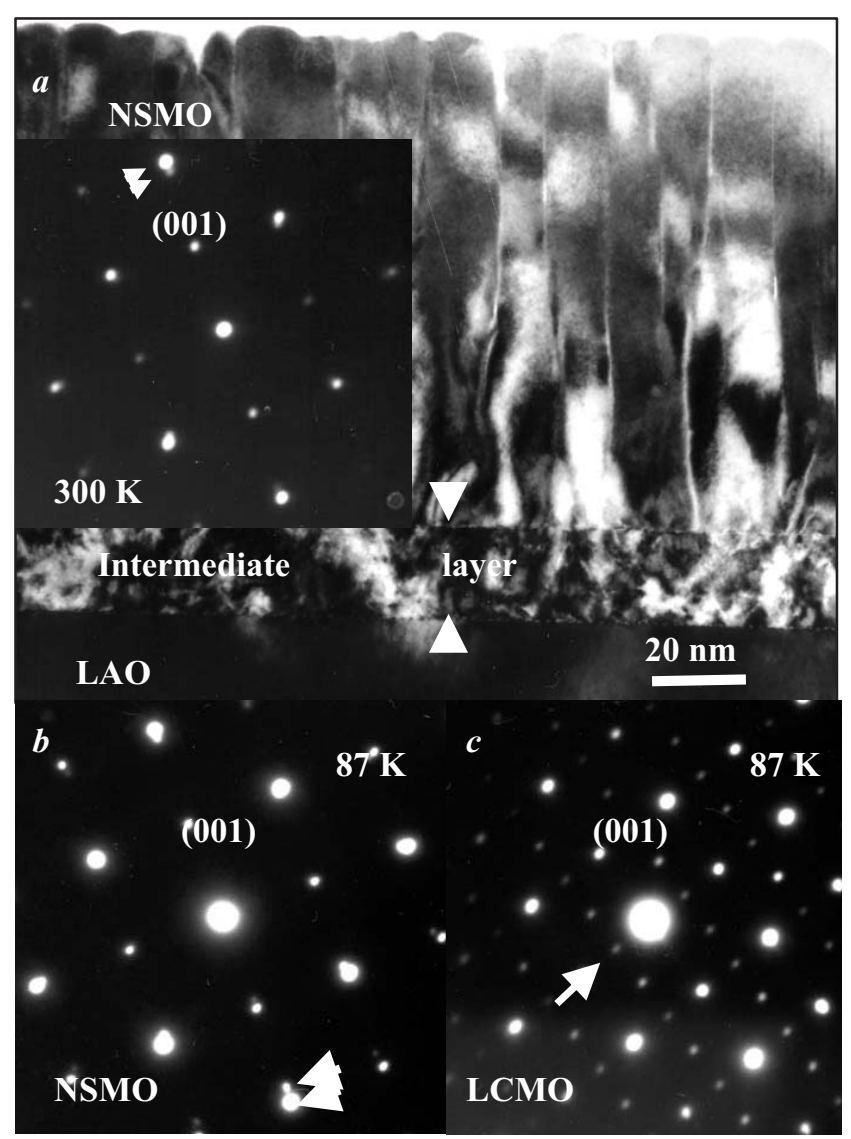

Fig. 2. $a-$ Low-magnification cross-sectional HREM image for the bilayered film across the interface with substrate measured at $300 \mathrm{~K}$. Inset shows the ED pattern taken at room temperature. $b-\mathrm{ED}$ pattern of the same film taken at $87 \mathrm{~K}$. $c$ - ED pattern taken at $87 \mathrm{~K}$ for the $\mathrm{La}_{0.5} \mathrm{Ca}_{0.5} \mathrm{MnO}_{3}$ film. White arrow indicates the superlattice reflections, connected with an appearance of the CO state.

slight splitting of the Bragg reflections along $c^{*}$ direction (indicated by white arrows) is observed for both temperatures. Therefore, the high- $T_{\text {sub }}$ film has an additional crystal phase with the out-of-plane lattice parameter, which is larger than that for the bulk, $c \simeq 0.3896 \mathrm{~nm}$. The existence of two phases with different crystal structure is well-defined on the cross-sectional low-magnification HREM image, taken at a room temperature and represented by the Fig. 2, $a$. It should be noted that the HREM image prepared at $87 \mathrm{~K}$ remains without changing. Analysis of the transmission contrast of the HREM image reveals that the film has a column-like microstructure (with the average diameter of the columns about $10 \mathrm{~nm}$ ) and the sufficiently thick $(\approx 20 \mathrm{~nm})$ intermediate layer on the film side (indicated by the white arrows). An additional FFT analysis (not shown) manifests that particularly the intermediate layer has a crystal lattice with the tetragonal distortion $(c / a \simeq 1.015)$, while the rest of the film with a column-like microstructure has lattice parameters coinci- dent to the bulk. Since the intermediate layer has a tetragonal distortion, one can assume that it is the slightly strained crystalline phase due to the large mismatch between film and substrate lattice parameters. On the other hand, as the thickness of grown layer exceeds a certain critical value during the film's deposition, the film tend to stress relaxation by the formation of the misfit dislocations or a columnlike microstructure directed normal to the film plane [14]. This film hereinafter will be referred as a self-organizing bilayered one.

The ED patterns of the high- $T_{\text {sub }}$ NSMO film, prepared at $300 \mathrm{~K}$ (inset in Fig. 2,a) and $87 \mathrm{~K}$ (Fig. 2,b), display only fundamental Bragg spots without traces of the CO phase, which is observed at $T_{\mathrm{CO}} \simeq 150 \mathrm{~K}$ in bulk as a rule. It is well-known that the $\mathrm{CO}$ phase is characterized by the appearance of superlattice spots on the ED patterns, with a wave vector $q=a^{*} / 2$, where $a^{*}$ is the reciprocal lattice vector along the $a$ axis for the simple pseudocubic symmetry. For comparison Fig. 2,c shows the same ED pattern for the half-doped $\mathrm{La}_{0.5} \mathrm{Ca}_{0.5} \mathrm{MnO}_{3}$ (LCMO) film, prepared at the same conditions, in which the CO state is observed [15]. The superlattice reflections are indicated by a white arrow. The nonappearance of the CO phase in the high- $T_{\text {sub }}$ NSMO film even at low temperature $(87 \mathrm{~K})$ can be explained by two following circumstances. First, the major part of the film consists the small-size columnlike clusters that provide the complete suppression of the CO phase [9]. Second, the intermediate layer has an in-plane compressive strained crystal lattice that also leads to a degradation of the CO state [5].

Summarizing the microstructural measurements one can conclude that three kinds of the $\mathrm{Nd}_{0.5} \mathrm{Sr}_{0.5} \mathrm{MnO}_{3}$ films with different microstructure were prepared. There are the amorphous $\left(T_{\text {sub }} \simeq 20^{\circ} \mathrm{C}\right)$, the nanoclustered $\left(T_{\text {sub }} \simeq 300{ }^{\circ} \mathrm{C}\right)$, and the self-organizing bilayered $\left(T_{\text {sub }} \simeq 750{ }^{\circ} \mathrm{C}\right)$ films. All films have a nano-crystalline microstructure and do not manifest the traces of the $\mathrm{CO}$ state with the decreasing temperature up to $87 \mathrm{~K}$.

\section{Experimental results}

Figure 3 shows both FC (solid symbols) and ZFC (open symbols) temperature-dependent magnetization curves, $M(T)$, at different magnetic field for the amorphous film. The $M(T)$ behavior is typical for paramagnetic (PM) materials [16] and could be written for the whole temperature range as

$$
M^{P M}(T, H)=\left[\chi_{0}+C_{C W} /(T+\theta)\right] H,
$$

where $\chi_{0}$ is a temperature-independent susceptibility, and the second term is the Curie-Weiss (CW)-type susceptibility with a constant $C_{C W}$ and a characteristic temperature $\theta$. Inset shows that the experimental data are excellently described by the $\mathrm{CW}$ expression with the following 


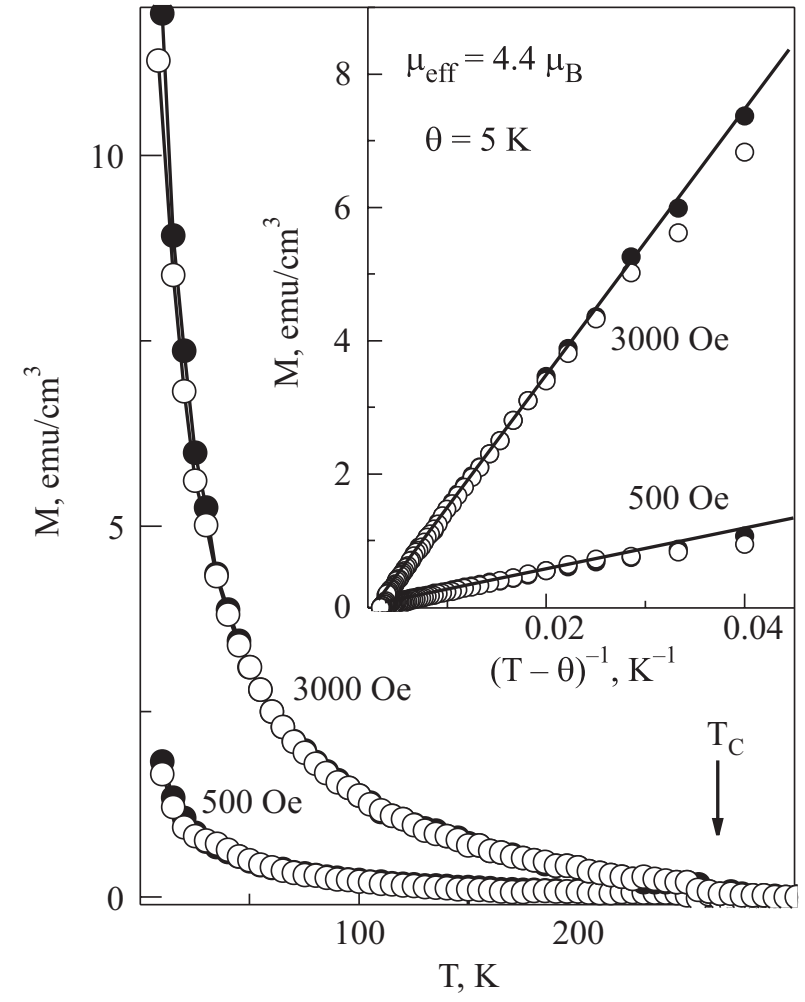

Fig. 3. Temperature dependence of the in-plane FC (solid symbols) and ZFC (open symbols) of a magnetization for the amorphous film measured under an applied magnetic field of 500 and 3000 Oe. Lines are guides to the eyes. Arrow indicates the location of the Curie temperature for this compound. Inset is the $M(T)$ versus $1 /(T-\theta)$ plot for the same film.

fitting parameters: $C_{C W} \simeq 0.002 \mathrm{emu} \cdot \mathrm{K} / \mathrm{T}$ and $\theta=5 \mathrm{~K}$. The estimated from $C_{C W}$ effective magnetic moment was $\mu_{\text {eff }} \simeq 4.4 \mu_{B} / \mathrm{Mn}$, that is almost coincident with the theoretical value, $\mu_{\text {eff }}^{\text {theor }} \simeq 4.42 \mu_{B} / \mathrm{Mn}$, which is obtained from following expression:

$$
\mu_{\mathrm{eff}}^{\text {theor }}=g \sqrt{x S_{1}\left(S_{1}+1\right)+(1-x) S_{2}\left(S_{2}+1\right)} .
$$

Here $x$ is the Sr concentration, $S_{1}=3 / 2$ and $S_{2}=2$ are the spin values of $\mathrm{Mn}^{4+}$ and $\mathrm{Mn}^{3+}$ ions, respectively, and $g=2$ is the Landé factor. Therefore, one can conclude that the amorphous film is a typical paramagnet with a freely moving of the individual $\mathrm{Mn}$ spins.

Figure 4 presents the $M(T)$ curves for the nanoclustered film measured under the different applied magnetic field. The magnetization value monotonically increases with decreasing temperature for all the applied fields, demonstrating the superparamagnetic (SPM) behavior up to about $40 \mathrm{~K}$. Moreover, the ZFC and FC $M(T)$ curves are nonsplitted in this temperature range. At the same time, the significant difference between ZFC and FC $M(T)$ is occurred below of $40 \mathrm{~K}$, indicating appearance of an additional magnetic transition. Inset shows the in-plane magnetization loops for this film at different

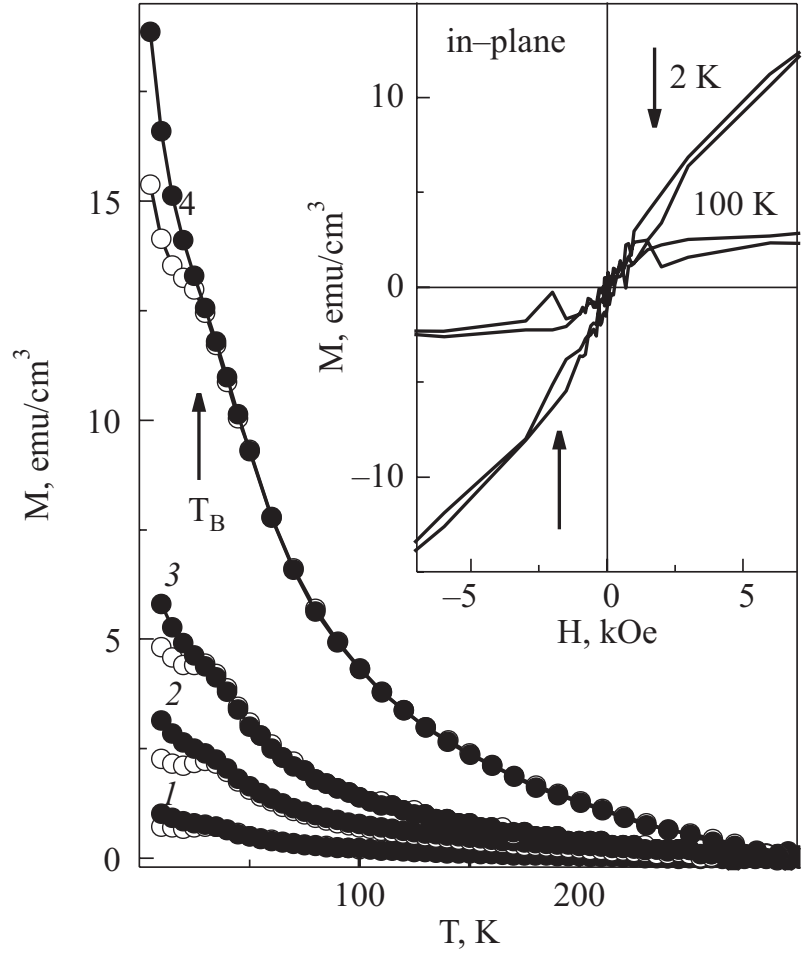

Fig. 4. Temperature dependence of the in-plane FC (solid symbols) and ZFC (open symbols) of a magnetization for the nanoclustered film measured under an applied magnetic field of 500 (1), 1500 (2), 3000 (3), and 10000 (4) Oe. Lines are guides to the eyes. Arrow indicates location of the blocking temperature. Inset shows the in-plane hysteresis loops at 2 and $100 \mathrm{~K}$. Arrows indicate the hysteretic lobes in a high-field range.

temperature. It is seen that the $M(H)$ dependences manifest a nonhysteretic behavior in the low-field range but have the hysteretic lobes (indicated by arrows) at higher magnetic field.

Figure 5 shows the temperature-dependent magnetization of the self-organizing bilayered film. The main feature of this film lies in the unique $M(T)$ behavior with increasing applied magnetic field. At low applied magnetic field (100 Oe) $M(T)$ demonstrates a shape, which is typical for the FM state (the magnetization tends to a saturation at low temperature), while at higher one the SPM-like behavior is added (the magnetization permanently grows with the decreasing temperature). Therefore, the $M(T)$ dependences testify for a presence of two different magnetic phases, FM and SPM, which are located, probably, in the different regions of the film. Taking into account the HREM analysis (Fig. 2,a), one can conclude that FM belongs to the intermediate layer while SPM occupies the film's part with the nanocrystalline column-like microstructure. Inset displays the in-plane magnetization loops for this film at different temperature. In contrast to the nanoclustered film the $M(H)$ dependence reveals the well-defined symmetric hysteretic loop at $T=$ $=100 \mathrm{~K}$, which confirms a presence of the FM phase. At 


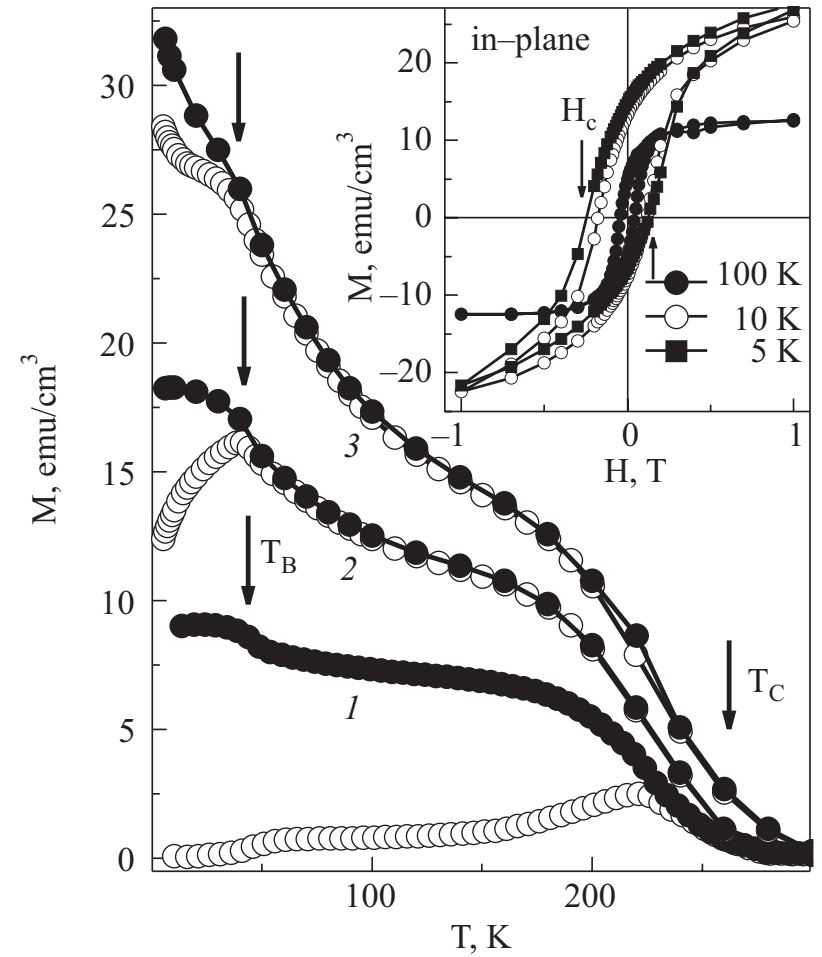

Fig. 5. Temperature dependence of the in-plane FC (solid symbols) and ZFC (open symbols) of a magnetization for the bilayered film measured under an applied magnetic field of 100 (1), 3000 (2), and 10000 (3) Oe. Lines are guides to the eyes. Arrows indicate location of the blocking temperature. Inset shows the in-plane hysteresis loops at different temperature. Arrows indicate the coercive field $H_{c}$.

the same time, with decreasing temperature up to $5 \mathrm{~K}$ the magnetization loops become greatly asymmetric. Similar to the nanoclustered film, the additional magnetic transition is observed below $50 \mathrm{~K}$ with decreasing temperature as well.

\section{Discussion}

Let us consider the peculiarity of the magnetic state in the $\mathrm{Nd}_{0.5} \mathrm{Sr}_{0.5} \mathrm{MnO}_{3}$ film controlled by a different kind of the microstructure disorder.

Figure 3 reveals that the amorphous film is a typical paramagnet with a freely moving of the individual $\mathrm{Mn}$ spins, the $M(T)$ dependence of which is well described in framework of the Curie-Weiss approximation. Therefore, the quench-disordered microstructure, which is formed during the deposition at $T_{\text {sub }} \simeq 20{ }^{\circ} \mathrm{C}$, results in the paramagnetic state of the film. Such effect have already observed by us in the $\mathrm{La}_{0.7} \mathrm{Ca}_{0.3} \mathrm{MnO}_{3}$ films [12] and can be explained by the anomalously small size of the crystallites.

In contrast to that the temperature dependence of a magnetization for the nanoclustered film, deposited at
$T_{\text {sub }} \simeq 300{ }^{\circ} \mathrm{C}$, cannot be described by the CW approximation. Figure 6 shows the $M(T)$ versus $1 / T$ plot for this film, measured at different applied magnetic fields, which does not demonstrate a linear behavior in the whole temperature range. Such a nonlinear behavior of $M(H / T)$ is more typical for the SPM particles and can be explained with the Langevin function [16]:

$$
M^{S P M}(T, H)=M_{S}^{S P M}\left[\operatorname{coth}\left(\frac{\mu_{\mathrm{eff}} H}{k_{B} T}\right)-\frac{k_{B} T}{\mu_{\mathrm{eff}} H}\right],
$$

where $M_{S}^{S P M}$ is the saturated magnetization of a SPM phase and $\mu_{\mathrm{eff}}$ is the average magnetic moment of the SPM particles. Solid lines in Fig. 6 represent fitted Langevin functions closest to the experiment data with a variation of the average effective magnetic moment, $\mu_{\mathrm{eff}}$. It is surprisingly that $\mu_{\text {eff }}$ greatly dependent on an applied magnetic field through the empirical function of $\mu_{\text {eff }} \sim 1 / H$. By taking the saturation magnetic moment for bulk $\mathrm{Nd}_{0.5} \mathrm{Sr}_{0.5} \mathrm{MnO}_{3}$ in the FM state as $3.5 \mu_{B} / \mathrm{Mn}$ [17] and assuming a spherical shape of the SPM clusters with a volume of $\pi D^{3} / 6$, we estimate their average diameter to be $D \simeq 4.3$ and $1.7 \mathrm{~nm}$ for 100 and 10000 , respectively. In principle, it is coincident with the ED data, particularly in the case of a lowest applied magnetic field, and allow us to conclude that the nanocrystalline disorder leads to formation of the SPM state in the film. However, the size of

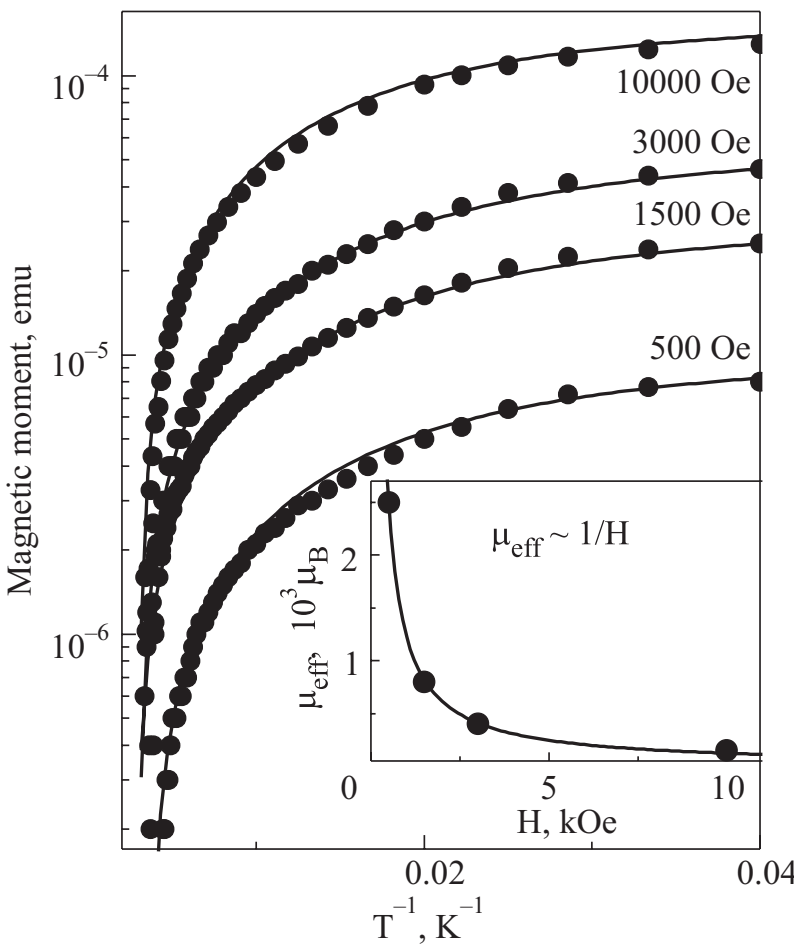

Fig. 6. $M(T)$ versus $1 / T$ plot for the nanoclustered film. Solid lines are represent fitted Langevin functions closest to the experiment data with a variation of the effective magnetic moment $\mu_{\text {eff }}$. Inset displays the $\mu_{\text {eff }}(H)$ dependence simulated on the base of the Langevin approximation. 
the SPM particle is not rigidly bound with the crystalline cluster and is controlled by an applied magnetic field, contradicting to the underlying principles of the theoretical models for an ensemble of the SPM particles. The similar unusual FC $M(T)$ behavior was observed already in the $\mathrm{La}_{1-x} \mathrm{Ca}_{x} \mathrm{MnO}_{3} \mathrm{FM} / \mathrm{AFM}$ multilayers and explained by the thermal instability of different exchange paths, first of all, a spin-flop transition in AFM grains under an applied magnetic field [18]. However, in our case both the FC and ZFC $M(T)$ curves demonstrate an unsplitted exponential behavior, beginning from the lowest applied magnetic field, except for the temperature range below $T_{B}$.

A following explanation on the unusual $M(H, T)$ behavior can be proposed. It is believable that the FM ground state in the manganites is provided by the Zener's double exchange interaction by means of an electron transfer from $\mathrm{Mn}^{3+}$ to $\mathrm{Mn}^{4+}$ via the oxygen [1]. Because any structural defects, such as grain or twin boundaries, for example, block up the electron motion, the FM coupling between the adjacent crystalline clusters will be also suppressed. Consequently, the individual crystallites in the nanoclustered film can be treated as the independent magnetic domains with the abnormally small interdomain separation. Such type of the magnetic objects lower their magnetostatic energy by forming the closure-domain [19] or the magnetic vortex [20] structure. Therefore, the nanocrystalline clusters in this film are not the FM single domains and their magnetic properties can not to be correctly described in the framework of a classical Langevin statistics, expressed by Eq. (1). It is confirmed by the unusual shape of the hysteresis loops represented by the inset in Fig. 4. The loops have hysteretic lobes (indicated by arrows) but are nonhysteretic as the field crosses zero. Such kind of the hysteresis loop was simulated recently for submicron Permalloy dot arrays, utilizing the concept of the magnetic vortex (MV) [21]. Therefore, one can conclude that the nanoclustered film demonstrates the magnetic properties, which are resemble the behavior for an ensemble of the SPM particles, however, each particle in this case must be consider as a closure magnetic domain (or magnetic vortex) rather than the single one.

Figure 4 displays that the FC and ZFC $M(T)$ curves become splitted at $T \leq T_{B}$. The similar effect is very often observed in an ensemble of FM (or AFM) nanoparticles and explained in framework of the Néel-Brown theory $[22,23]$. According to this model, there is a critical temperature, called the blocking one, given by $T_{B}=$ $=E_{A} /\left[\ln \left(\tau_{\text {obs }} / \tau_{0}\right) k_{B}\right]$, above which the FM nanoparticles transform from the blocked to unblocked SPM state. Here $E_{A}$ is the energy barrier between two directions of the magnetic moment in a single domain nanoparticle — along and against to an applied magnetic field, $\tau_{\text {obs }}$ is the observation time, and $\tau_{0}$ is the characteristic time constant, called the attempt frequency. On the other hand, the magnetic vortex also has two different magnetic states separated by an energy barrier, owing to two key properties: chirality (clockwise or counterclockwise) and polarity (positive or negative) [20]. It is reasonable to suggest that below a certain temperature these states can be blocked (or frozen) if the thermal energy becomes smaller than the energy barrier between different states of the magnetic vortex. Therefore, the observed splitting between the FC and ZFC $M(T)$ curves, represented by Fig. 4, can be treated as a blocked-unblocked phase transition in an assembly of the SPM particles, and $T_{B}$ is the blocking temperature. However, it worth to note, that in contrast to the usual FM single domain nanoparticles the $M(H)$ dependence measured at $T<T_{B}$ (see inset) manifests the nonhysteretic loop as the magnetic field crosses zero.

The self-organizing bilayered film, taking into account a microstructure analysis, consist of the two layers with the different microstructure and the same chemical composition. The temperature-dependent magnetization curve in this case can be represented by superposition of the FM and SPM terms: $M(T)=M^{F M}(T)+M^{S P M}(T)$. The $M^{F M}(T)$ term belongs to the intermediate layer with the perfect (but slightly strained) crystal structure while $M^{S P M}(T)$ defines the magnetic properties of major part of the film with the column-like structure. Figure 5 shows that the intermediate layer undergoes only the FM transition at $T_{C} \simeq 250 \mathrm{~K}$ with decreasing temperature, which is good coincident with the published results. At the same time, the part of the film with the column-like structure demonstrates the $M^{S P M}(T)$ behavior similar to that was observed for the nanoclustered one (see Fig. 4). The exponential growth of the $M^{S P M}(T)$ with decreasing temperature is ended by the well-defined FC-ZFC splitting at the high applied magnetic field (curves 2 and 3) or the kink-like peculiarity when $H$ is smaller of the coercive field $H_{c}$ (curve 1). Inset in Fig. 5 reveals that $H_{c} \simeq$ $\simeq \pm 450 \mathrm{Oe}$ at $100 \mathrm{~K}$, which is larger than an applied magnetic field taken for measure of the curve $1(H=100 \mathrm{Oe})$. Therefore, the observed large difference between the FC and ZFC $M(T)$ experimental curves, starting closely below $T_{C}$, concerns to the FM transition only in the intermediate layer and can be explained by the lattice distortion [14]. Therefore, the self-organizing bilayered film can be treated as a hybrid magnetic system involving the SPM and FM layers with a very sharp interface (see Fig. 2). The blocking temperature in the this film $\left(T_{B} \simeq 50 \mathrm{~K}\right)$ turn out to be slightly larger than that observed in the nanoclustered one $\left(T_{B} \simeq 40 \mathrm{~K}\right)$. We assume that the observed increase in $T_{B}$ is governed by an additional exchange coupling between the SPM and FM layers which 
leads to increase of the energy barrier between different state of the SPM particle.

Inset in Fig. 5 displays that the hysteresis loop of the bilayered film greatly changes the shape with decreasing temperature. It is seen the hysteresis loop is symmetrical at $100 \mathrm{~K}$ with the coercive field $H_{\mathrm{c}} \simeq \pm 450$ Oe while becomes greatly asymmetric at 10 and $5 \mathrm{~K}, H_{c} \simeq+1200$ and -1800 , and +1250 and $-2500 \mathrm{Oe}$, respectively. This effect is observed as a rule in the FM/AFM hybrid system and explained by the appearance of an exchange-bias interaction on the interface between the FM and AFM phases [24]. Exchange bias is a measure of the shift in the hysteresis loop along the applied magnetic field axis of FM when it is magnetically coupled to AFM [25]. This is commonly characterized by an exchange-bias field $H_{E B}$ through which the center of the hysteresis loop of FM is shifted from zero. In our case $H_{E B} \simeq-300$ and -625 Oe at $T=10$ and $5 \mathrm{~K}$, respectively. Therefore, the self-organizing bilayered film demonstrates a negative exchange bias which is typical for the FM(particles)/AFM(layer) hybrid system in spite of that we deal with the FM(particles)/FM(layer) one, in which this phenomenon is quite unexpected. We suggest that the found contradiction is connected with the closure-domain (or magnetic vortex) nature of the nanoclusters which play a role of the SPM particles in our film. In this case the SPM particle, forming the magnetic vortex, can be treated as the AFM rather than FM domain. Therefore, in fact, the bilayered film represents the AFM(particles)/ FM(layer) hybrid system. At high temperature $\left(T \geq T_{B}\right)$ the AFM-like domains have a behavior, which is similar to the freely moving SPM particles without any interaction with FM intermediate layer. In this case the hysteresis loop is symmetrical (see the inset in Fig. 5). When a temperature decreases, $T \leq T_{B}$, the AFM-like domains transform in the blocking state and induce exchange anisotropy in the FM layer which shifts the hysteresis loop towards negative $H$ values. Moreover, the exchange bias field $H_{E B}$ greatly increases with decreasing temperature that absolutely agrees with published results for the AFM/FM layered systems.

\section{Conclusions}

In summary, the amorphous, the nanoclustered, and the self-organizing bilayered films have been prepared by the rf-magnetron sputtering using a so-called «soft» (or powder) target. The amorphous film turn out to be a typical paramagnet with a freely moving of the individual Mn spins, the magnetic properties of which is well described in framework of the Curie-Weiss approximation. The nanoclustered film manifests the magnetic properties mimic to the ensemble of the SPM particles, however, which can not be correctly described in the framework of a classical Langevin approach. Taking into account, the unique shape of the hysteresis loops, which have hysteretic lobes at high magnetic field but are nonhysteretic as the field crosses zero, we suggest that each particle is a magnetic vortex rather than the single domain. At the same time, the unblocked to blocked transition was observed in the film with decreasing temperature. This testify that the magnetic vortex, similar to the single-domain particle, can transfer from a high-temperature SPM to a low-temperature blocked (or «pinned») state at $T \leq T_{B}$. The self-organizing bilayered film demonstrates a negative exchange bias, which is typical for the FM/AFM hybrid system. The exchange bias interaction leads to the increase of $T_{B}$, the significant shift in the hysteresis loop along the applied magnetic field axis, $H_{E B} \simeq-625$ Oe at $5 \mathrm{~K}$, and the broadening of a hysteresis loop. All films did not manifest the charge ordering in the whole temperature range up to $87 \mathrm{~K}$.

This work was supported by the KOSEF through the Quantum Photonic Science Research Center.

1. For a review, see Colossal Magnetoresistance, Charge Ordering and Related Properties of Manganese Oxides, C.N.R. Rao and B. Raveau (eds.), World Scientific, Singapore (1998); Colossal Magnetoresistance Oxides, Y. Tokura (ed.), Gordon and Breach, London (1999); E. Dagotto, T. Hotta, and A. Moreo, Phys. Rep. 344, 1 (2001).

2. R. Kajimoto, H. Yoshizawa, H. Kawano, H. Kuwahara, Y. Tokura, K. Ohoyama, and M. Ohashi, Phys. Rev. B60, 6506 (1999).

3. T. Fujiwara and M. Korotin, Phys. Rev. B59, 9903 (1999).

4. Y. Ogimoto, M. Nakamura, N. Takubo, H. Tamaru, M. Izumi, and K. Miyano, Phys. Rev. B71, 060403 (2005).

5. H. Wang, X. Zhang, M.F. Hudley, J.D. Thomoson, B.J. Gibbons, Y. Lin, P.N. Arendt, S.R. Foltyn, Q.X. Jia, and J.L. MacManus-Driscoll, Appl. Phys. Lett. 84, 1147 (2004).

6. Q. Qian, T.A. Tyson, C.-C. Kao, W. Prellier, J. Bai, A. Biswas, and R.L. Greene, Phys. Rev. B63, 224424 (2001).

7. C. Cui, T.A. Tyson, Z. Chen, and Z. Zhong, Phys. Rev. B68, 214417 (2003).

8. R.C. Yu, J. Tang, L.D. Yao, A. Matsushita, Y. Yu, F.Y. Li, and C.Q. Jin, J. Appl. Phys. 97, 083910 (2005).

9. P. Levy, F. Parisi, G. Polla, D. Vega, G. Leyva, H. Lanza, R.S. Freitas, and L. Ghivelder, Phys. Rev. B62, 6437 (2000).

10. V. Ponnambalam, S. Parashar, A.R. Raju, and C.N.R. Rao, Appl. Phys. Lett. 74, 206 (1999).

11. V.G. Prokhorov, G.G. Kaminsky, V.A. Komashko, J.S. Park, and Y.P. Lee, J. Appl. Phys. 90, 1055 (2001).

12. V.G. Prokhorov, V.A. Komashko, G.G. Kaminsky, V.L. Svetchnikov, Y.P. Lee, and S.Y. Park, Fiz. Nizk. Temp. 30, 938 (2004) [Low Temp. Phys. 30, 705 (2004)].

13. P.M. Woodward, D.E. Cox, T. Vogt, C.N. R. Rao, and A.K. Cheetham, Chem. Mater. 11, 3528 (1999).

14. Y.P. Lee, S.Y. Park, Y.H. Hyun, J.B. Kim, V.G. Prokhorov, V.A. Komashko, and V.G. Svetchnikov, Phys. Rev. B73, 224413 (2006). 
15. V.G. Prokhorov, V.A. Komashko, G.G. Kaminsky, Y.P. Lee, S.Y. Park, Y.H. Hyun, V.L. Svetchnikov, K.W. Kim, and J.Y. Rhee, Low Temp. Phys. 32, 128 (2006).

16. B.D. Cullity, Introduction to Magnetic Materials, Addison-Wesley, New York (1972).

17. J. Geck, D. Buns, C. Hess, R. Klingeler, P. Reutler, M.v. Zimmermann, S.-W. Cheong, and B. Büchner, Phys. Rev. B66, 184407 (2002).

18. N. Moutis, C. Christides, I. Panagiotopoulos, and D. Niarchos, Phys. Rev. B64, 094429 (2001).

19. C. Kittel, Introduction to Solid State Physics, 7th ed. Wiley, New York (1996).
20. V. Novosad, K. Yu. Guslienko, H. Shima, Y. Otani, S.G. Kim, K. Fukamichi, N. Kikuchi, O. Kitakami, and Y. Shimada, Phys. Rev. B65, 060402 (2002).

21. K.Yu. Guslienko, V. Novosad, Y. Otani, H. Shima, and K. Fukamichi, Appl. Phys. Lett. 78, 3848 (2001).

22. L. Néel and C.R. Acad. Sci. URSS 228, 664 (1949).

23. W.F. Brown, Phys. Rev. 130, 1677 (1963).

24. W.H. Meilejohn and C.P. Been, Phys. Rev. 102, 1413 (1956).

25. For a review, see J. Nogués and I.K. Schuller, J. Magn. Magn. Mater. 192, 203 (1999). 